\title{
Relating Function to Branching Geometry: A Micro-CT Study of the Hepatic Artery, Portal Vein, and Biliary Tree
}

\author{
Timothy L. Kline ${ }^{\mathrm{a}}$ Mair Zamir ${ }^{\mathrm{b}} \quad$ Erik L. Ritman ${ }^{\mathrm{a}}$ \\ ${ }^{a}$ Department of Physiology and Biomedical Engineering, Physiological Imaging Research Laboratory, \\ Mayo Clinic, College of Medicine, Rochester, Minn., USA; ${ }^{b}$ Departments of Applied Mathematics and of \\ Medical Biophysics, The University of Western Ontario, London, Ont., Canada
}

\section{Key Words}

Anatomy, functional $\cdot$ Blood vessel, morphology and structure $\cdot$ Morphology, comparative $\cdot$ Liver $\cdot$ Rat

\begin{abstract}
Utilizing micro-computed tomography images, the hierarchical structure, interbranch segment lengths and diameters of a hepatic artery, a portal vein, and two biliary trees from intact rat liver lobes were characterized. The data were investigated by analyzing the geometric properties of the vascular structures, such as how interbranch segment diameters change at bifurcation points. In the case of the hepatic artery and portal vein trees (in which the flow rate is high by comparison with that in the biliary tree), the vascular geometry is consistent with a fluid transport system which aims to simultaneously minimize both the power loss of laminar flow, and a cost function proportional to the total volume of material needed to maintain the system (lumenal contents). In comparison, the biliary tree (which has a low flow rate and an opposite flow direction to that of the hepatic artery and portal vein) was found to have a geometry in which the lumen cross-sectional area is maintained at bifurcations. These findings imply that the histological makeup and therefore the pathophysiology of biliary tree vasculature are likely very different from that of the vasculature within the systemic ar-
\end{abstract}

\section{KARGER}

Fax +4161306 1234

E-Mail karger@karger.ch

www.karger.com (c) 2011 S. Karger AG, Basel

Accessible online at: www.karger.com/cto terial tree. The extent to which the characteristic variability/ scatter in the data may have resulted from imaging and/or measurement errors was examined by simulating such errors in a theoretical tree model and comparing the results with the measured data.

Copyright $\odot 2011$ S. Karger AG, Basel

\section{Introduction}

The liver, a wedge-shaped organ, is the largest gland in the body [Standring and Gray 2008]. It is $\sim 4 \%$ of an adult rat's total body weight $(\sim 300 \mathrm{~g})$, receiving around $12 \mathrm{ml}$ of blood per minute [Birnie and Grayson, 1952]. As a reference, an average human's liver is around $2.5 \%$ of their total body weight and hepatic blood flow is around 1,500 $\mathrm{ml} / \mathrm{min}$ [Sinnatamby, 2006]. The liver has two surfaces, the 'diaphragmatic surface' and the 'visceral surface'. The

\section{Abbreviations used in this paper}

3D 3-dimensional

micro-CT micro-computed tomography 


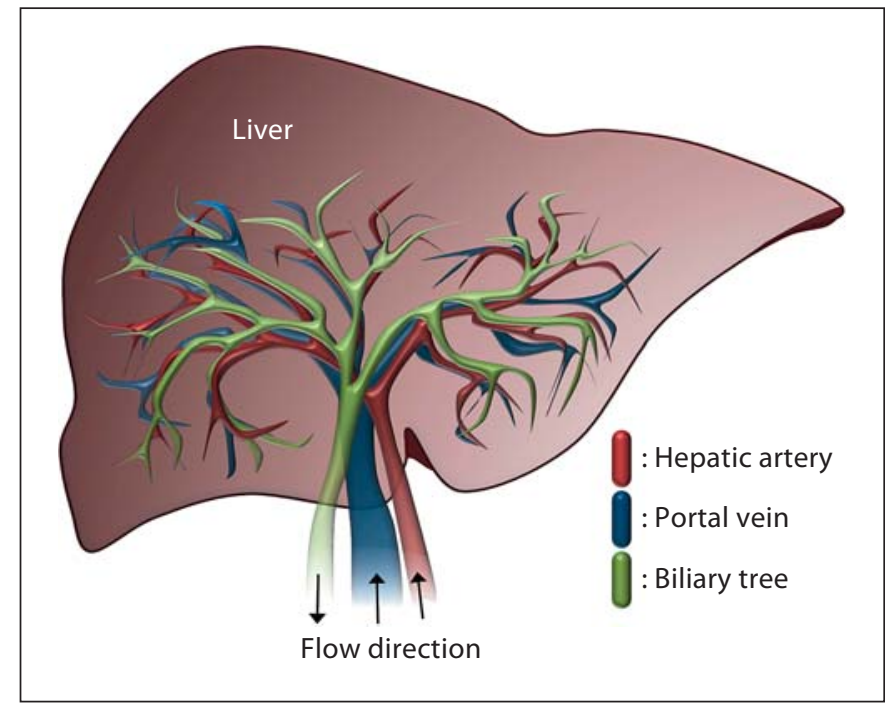

Fig. 1. The hepatic artery, portal vein, and biliary tree all follow roughly the same anatomical paths throughout the liver, and all three enter the liver through the porta hepatis. The liver outline is also depicted for orientation.

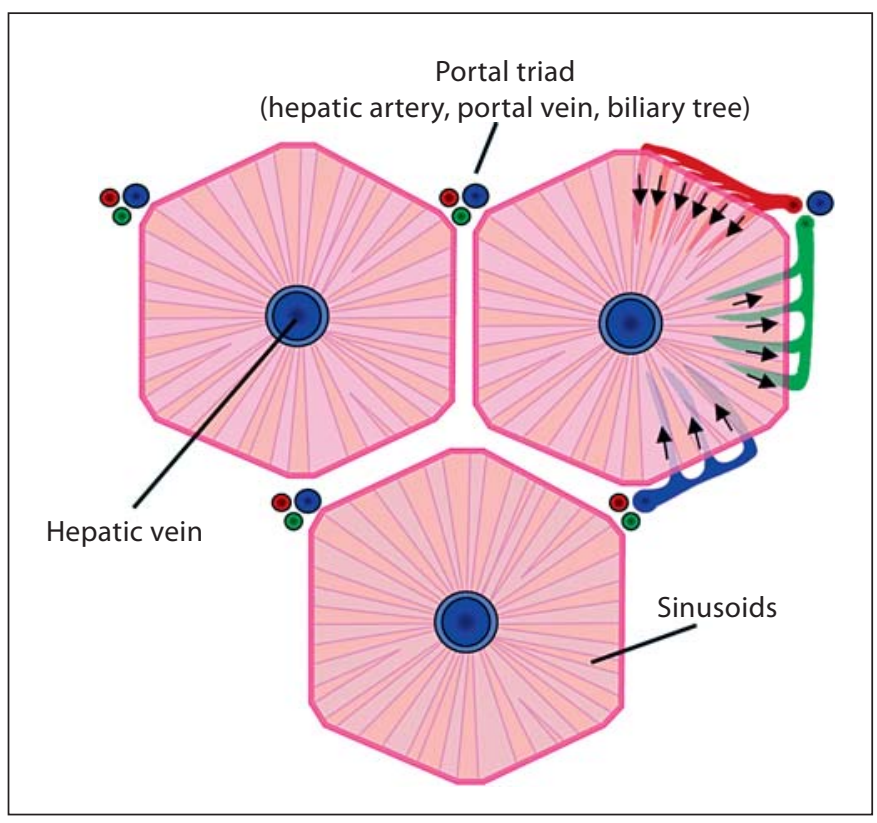

Fig. 2. The liver lobules are a roughly hexagonal arrangement of hepatocyte plates which are separated by intervening sinusoids (small blood vessels similar to capillaries but with a fenestrated endothelium) which radiate outward from a central vein. The portal triads are located at the vertices of each hexagon. The different functions of the terminal branches of the hepatic artery, portal vein, and biliary tree occur within each lobule. A central vein drains each lobule, which is carried to the hepatic vein away from the liver. diaphragmatic surface is convex and is moulded to the undersurface of the diaphragm. The porta hepatis, a deep fissure on the visceral surface, is where the hepatic artery and portal vein enter, and the biliary tree exits the liver.

At the porta hepatis, two layers of the lesser omentum enclose the right and left hepatic ducts (components of the biliary tree), as well as the right and left branches of the hepatic artery and portal vein. A diagram of the relevant liver vasculature for this study is shown in figure 1. The flow direction of the hepatic artery, portal vein, and biliary tree is shown, along with the liver surface for orientation. The vascular structures lie in the order of portal vein, hepatic artery, and bile ducts (therefore bile ducts are more accessible during surgery due to being located most ventral of the three [Sinnatamby, 2006]).

Oxygenated blood to the liver is provided by the hepatic artery. Upon entering the liver at the porta hepatis, the hepatic artery divides into right and left branches. The hepatic artery supplies $\sim 25 \%$ of the liver's total blood flow, while the remaining $\sim 75 \%$ is supplied by the portal vein. The portal vein is the upward continuation of the superior mesenteric vein. The portal vein carries venous blood to the liver, blood which contains the products of digestion which have been absorbed from the alimentary canal and are to be metabolized by liver cells. Like the hepatic artery, the portal vein also divides into right and left branches within the porta hepatis. The three hepatic ducts (right, left, and common), as well as the bile duct make up the extrahepatic biliary tract (note: rats do not have a gallbladder). The right and left hepatic ducts join to form the common hepatic duct at the end of the porta hepatis. Functionally, the biliary tree is the path by which bile is secreted throughout the liver and conveyed via tributaries down to a single duct that enters the small intestine. A potential consequence of the different flow direction in the biliary tree is that in addition to flow magnitude, local shear stress distribution pattern asymmetries in converging and diverging flows may affect local diameters [LaBarbera, 1995].

The hepatic artery, portal vein, and biliary tree follow essentially the same anatomical paths within the liver, thereby known collectively as the portal triad. The structural unit of the liver is the hepatic lobule. The hepatic lobules are a roughly hexagonal arrangement of hepatocyte plates, which are separated by intervening sinusoids (small blood vessels similar to capillaries but with a fenestrated endothelium) which radiate outward from a central hepatic vein. The portal triads are located at the vertices of each hexagon. A schematic depiction is given in figure 2. The functions of the terminal branches of the 
hepatic artery, portal vein, and biliary tree occur within each lobule. A central vein drains each lobule, which is carried to the hepatic vein away from the liver.

The liver is a relatively homogeneous organ possessing fewer anatomical constraints on vascular branching than, for instance, the constraints imposed on the coronary arteries and veins, with their different epicardial and intramyocardial environments, as well as on the pulmonary arteries and veins which are constrained by the bronchi. In addition, heart and lungs cyclically deform, meaning that their shape drastically changes within a short amount of time. Thus, vascular image 'snapshots' may not represent the underlying vascular geometry accurately. Therefore, liver vasculature offers an excellent opportunity to study the relationship between branching geometry and the functional roles of vasculature: in particular, the hepatic artery, portal vein, and biliary tree which are in close proximity to one another, and follow essentially the same branching paths (yet perform different functions).

The study of vascular branching geometry is an area of anatomy which has yet to reach the same level of accuracy and understanding as other areas (e.g., the macroscopic structure and physiological function of organs). The main reason for this has been the methodological difficulties in obtaining accurate data, and in building a cumulative understanding based on these data. The measurement of length and diameter, and the connectivity relationship between interbranch segments lie at the foundation of most vascular geometry studies [Suwa et al., 1963; Zamir, 1998; Op Den Buijs et al., 2006; Kassab, 2006]. In a previous study, Kline et al. [2010b] used optical measurement of the branches of intact casts of vascular trees as the gold standard, with each vascular segment mapped out and measured using micrometer translation and rotation. Based on these optical measurements, the tree's hierarchical structure in terms of interbranch segment connectivity, individual branch segment lengths, and individual branch segment diameters were established. The accuracy by which 3-dimensional (3D) micro-computed tomography (micro-CT) image data could be used to measure the vascular branching geometry, as compared to direct optical measurement (an established, but very laborious methodology) of vascular trees, was then characterized. This automated micro-CT image analysis method is used in the present study to analyze the different vascular branching geometries of the vessel tree. It was previously found that, on average, vessel segment diameters can be accurately measured to within 1 voxel $(19 \mu \mathrm{m})$. The absolute difference and relative error
Table 1. The median absolute difference and median relative error between micro-CT-measured values and those obtained by optical measurement at different diameter ranges

\begin{tabular}{lll}
\hline Diameter & $\begin{array}{l}\text { Absolute } \\
\text { difference, } \mathrm{mm}\end{array}$ & $\begin{array}{l}\text { Relative error } \\
\%\end{array}$ \\
\hline $0.30-0.80 \mathrm{~mm}$ & 0.015 & 3.1 \\
$0.20-0.29 \mathrm{~mm}$ & 0.020 & 7.4 \\
$0.10-0.19 \mathrm{~mm}$ & 0.017 & 10.7 \\
$0.05-0.09 \mathrm{~mm}$ & 0.013 & 15.8 \\
$0.00-0.04 \mathrm{~mm}$ & 0.009 & 20.6 \\
\hline
\end{tabular}

This data is from a previous study performed by the authors in which the accuracy of micro-CT image-based measurements of vascular branching geometry was determined [Kline et al., 2010b].

between the micro-CT measured value and the optical measurement at different diameter ranges is given in table 1 for reference [data from Kline et al., 2010b]. The extent to which the characteristic variability/scatter in the data may have resulted from imaging and/or measurement errors is examined in this current study by simulating such errors in a theoretical tree model and comparing the results with the measured data.

To investigate the branching geometry of the vascular trees, we analyzed micro-CT images of rat livers after contrast medium was injected into the vessel trees. The hierarchical structure, interbranch segment lengths, and interbranch segment diameters were characterized. These data were then investigated by analyzing the geometric properties of the vascular structures, in particular with regard to the model derived by Murray [Murray, 1926] on the basis that the fluid transport system (vasculature) geometry is consistent with simultaneous minimization of both the power loss of laminar flow, and the total volume of material needed to maintain the system (i.e., the lumenal contents). Note that these factors have opposing geometrical consequences.

\section{Materials and Methods}

\section{Specimens}

Previously prepared specimens [Masyuk et al., 2003] were used as the data set in this study, and are here briefly described. Male Fisher-344 rats weighing between 200 and 220 g were separated into three groups. Microfil, a radiopaque liquid silicone polymer compound (MV-122; Flow Tech, Inc., Carver, Mass., USA), was injected into either the hepatic artery (group 1), the 
biliary tree (group 2), or the portal vein (group 3). Therefore, a comparison of the different vessel structures necessitates the use of three different rat specimens: a hepatic artery from one, a biliary tree from another, and a portal vein from the last specimen. Two biliary tree specimens were analyzed to check for reproducibility of the results.

For the biliary tree injection, the infusion rate was between 0.01 and $0.05 \mathrm{ml} / \mathrm{min}$ and at a pressure of $7-10 \mathrm{~mm} \mathrm{Hg}$. For the hepatic artery, the infusion rate was $2 \mathrm{ml} / \mathrm{min}$ and at a pressure of $60-70$ $\mathrm{mm} \mathrm{Hg}$. For the portal vein, the infusion rate was $8-10 \mathrm{ml} / \mathrm{min}$ and at a pressure of $10-12 \mathrm{~mm} \mathrm{Hg}$. These infusion rates and pressures were chosen because they did not exceed the physiological flow and pressure in bile and blood of normal rats. Note that this particular Microfil compound has a viscosity of $25 \mathrm{cP}$, whereas blood has a viscosity of 3-4 cP. It is important to note that the viscosity of the Microfil compound is designed specifically to produce a desired rate of filling rather than to match the viscosity of blood. The role played by viscosity in the two cases is quite different.

After the vessels were perfused with Microfil, the specimens were stored under refrigeration overnight to allow polymerization. The following day the liver was dissected into individual lobes and immersed in a $10 \%$ buffered formalin solution. In order to dehydrate the liver, each lobe was placed in progressively higher concentrations of glycerin in water solutions (from 30 to 75\%) at 24-hour intervals, and then embedded in a clear BioPlastic polymer (Aldon Corp., Avon, N.Y., USA).

\section{Micro-CT Imaging}

The micro-CT imaging methods that were used to scan the specimens are discussed in more detail elsewhere [Jorgensen et al., 1998]. Briefly, the liver lobe was mounted on a computer-controlled rotating stage so that an X-ray projection image was generated in a cesium iodine crystalline plate, which converted the $\mathrm{X}$ ray into a light image, at each of 360 angles of view around $360^{\circ}$. This light image generated by the X-ray within the crystal plate was optically projected (and magnified) onto a charge-coupled device imaging array which converted the light intensity on each of the $1,024 \times 1,024,24 \mu \mathrm{m}$ on-a-side square pixels in the array to an electronic signal proportional to the light (X-ray) intensity. The scan data, once recorded, were normalized for the exposing $\mathrm{X}$-ray intensity and then subjected to a modified Feldkamp cone beam reconstruction algorithm [Feldkamp et al., 1984] to generate a $3 \mathrm{D}$ volume data set. The $3 \mathrm{D}$ image consisted of up to $1,024^{3}$ cubic voxels, each $\sim 20 \mu \mathrm{m}$ to a side, with gray scale proportional to the X-ray attenuation coefficient.

\section{Segmentation}

A binary file was computed by thresholding the gray scale $3 \mathrm{D}$ micro-CT images. The vascular trees were extracted from this image by means of a region-growing segmentation method to include those voxels, with gray scale values above the thresholded range, that were connected to the vessel tree's root (identified by visual inspection of the displayed 3D image). In order to include small vessel segments, a low CT threshold (much lower than half the maximum value found in large vessel lumens) was chosen, just above the 'noise' in the liver parenchyma in the CT image, in order to include vessel segments that had a reduced peak gray scale value caused by the modulation transfer function (blurring) of the micro-CT system. Specifically, the gray scale within a large vessel lumen is measured, as well as the gray scale in the surrounding tissue. The threshold is then set no higher than the average between these two values. The region-growing method resulted in a single connected tree structure for all specimens. These segmented trees, for all specimens, are displayed in figure 3 using the Analyze software package [Robb et al., 1989]. Shown in the upper left is the hepatic artery, the portal vein is in the upper right, the biliary tree (specimen $b t 1$ ) is in the lower left, and the second biliary tree specimen $(b t 2)$ is in the lower right.

\section{Vascular Tree Measurements}

The 3D segmented data set was used as the input for the extraction of the vascular tree's centerline ('skeleton'). The extraction of the vascular tree centerline allows for the simplification of the complex tree structure from which the branch segment location within the tree, and its connection to the other branch segments, is conveyed (essentially the first step in defining the vascular tree topology). Using locations along the centerline within the segmented image, the tree's hierarchical nature, branch lengths, and branch diameters were determined. More details on the centerline extraction method, and subsequent measurements of interbranch segment lengths and diameters can be found in Kline et al. [2009, 2010a, b].

\section{Characterization of Branching Geometry}

To further characterize the branching geometry of the different samples, we note that the vessel tree structures can be modeled as binary trees, where at each bifurcation one parent segment divides into two branches. By convention [Zamir, 2005], the 'level' (or generation) of the branches is one higher than that of their parent branch segment. The diameter of the branches $\left(d_{1}\right.$ and $\left.d_{2}\right)$ is related to the parent segment's diameter $\left(d_{0}\right)$ by

$$
d_{0}^{k}=d_{1}^{k}+d_{2}^{k}
$$

where $k$ is the junction exponent and under the framework of Murray's cube law $k=3$. To characterize the parameters of vascular branching, we computed properties such as the asymmetry ratio:

$$
\lambda=\frac{d_{2}}{d_{1}}, \quad d_{2} \leq d_{1},
$$

which has a convenient range lying between 0 and 1 . In nondimensional form the two branches are related to the asymmetry ratio by:

$$
\frac{d_{1}}{d_{0}}=\frac{1}{\left(1+\lambda^{k}\right)^{1 / k}}, \quad \frac{d_{2}}{d_{0}}=\frac{\lambda}{\left(1+\lambda^{k}\right)^{1 / k}},
$$

which shows the effects of relative vessel diameter. Also, the area ratio at an arterial bifurcation can be defined as:

$$
\beta=\frac{d_{1}^{2}+d_{2}^{2}}{d_{0}^{2}}=\frac{1+\lambda^{2}}{\left(1+\lambda^{k}\right)^{2 / k}},
$$

which shows how the area ratio depends on the degree of asymmetry as well as the power law index $k$.

To measure $k$, the method presented by Zamir et al. [1992] was used. Under this method, the relative error:

$$
e(k)=\frac{d_{0}^{k}-\left(d_{1}^{k}+d_{2}^{k}\right)}{d_{0}^{k}}
$$


Fig. 3. Volume renderings of the hepatic artery (a) and portal vein (b), biliary tree specimen $b t 1$ (c) and $b t 2$ (d) of rat liver specimens. These micro-CT data sets are here visualized with the Analyze software program [Robb et al., 1989].

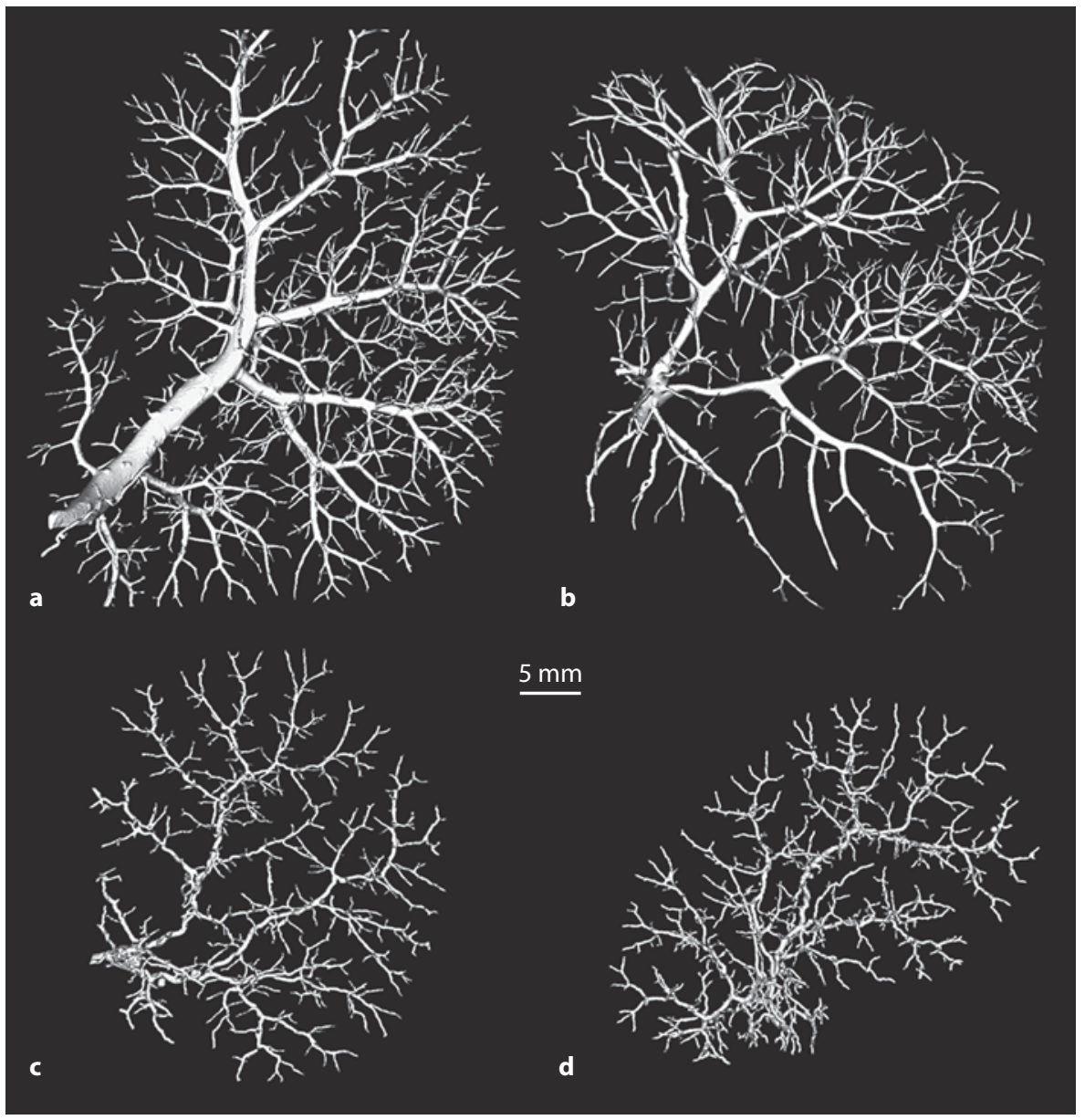

is computed for each junction. The median value of the error was computed for each vascular tree for junction exponents of $1,2,3$ and 4 . A linear fit of $k$ versus $e(k)$ has a y-intercept equal to the 'best-fit' junction exponent.

Finally, the power requirement or cost of deviating from the optimal configuration (i.e., $k=3$ ) was calculated using a formula presented by Sherman et al. [1989]:

$$
\psi=\frac{d_{0}^{-4}\left(d_{1}^{3}+d_{2}^{3}\right)^{4 / 3}+2 d_{0}^{2}\left(d_{1}^{3}+d_{2}^{3}\right)^{-2 / 3}}{3} .
$$

The basis for this equation is that we assume the daughter diameters are correct and the parent vessel's deviation from the optimal configuration results in the energy cost $\psi$. It is derived from the total power required to support both flow and metabolism.

\section{Effect of Variability}

The question of whether the observed differences between the biliary tree and the hepatic artery and portal vein could result from either variability in an ideal Murray-type tree, or measurement error, was explored with a theoretical tree model consisting of 10 generations and following Murray's law:

$$
d_{0}^{3}=d_{1}^{3}+d_{2}^{3} .
$$

Furthermore, we let the asymmetry ratio vary between generations as in:

$$
\lambda(j)=0.2 j-0.1(j-1),
$$

where $j$ represents the generations. Note that this criterion was chosen in order to consider a distribution of asymmetry ratios as is commonly found in physiological systems. We then incorporate a uniformly distributed random variability, in which the actual vessel segment diameter varies from the ideal configuration by

$$
d_{a}=d_{i}+R_{x} d_{i}
$$

where $d_{i}$ is the ideal Murray tree diameter, $R_{x}$ is the random variable multiplier, and $d_{a}$ is the modified diameter. As an example, when the variability is set at $5 \%$, the random variable lies within the range between -0.05 and +0.05 .

\section{Results}

Displayed in figure 4 are the results of the average vessel segment diameter versus generation, for the hepatic artery (fig. 4a), the portal vein (fig. 4b), the biliary tree 


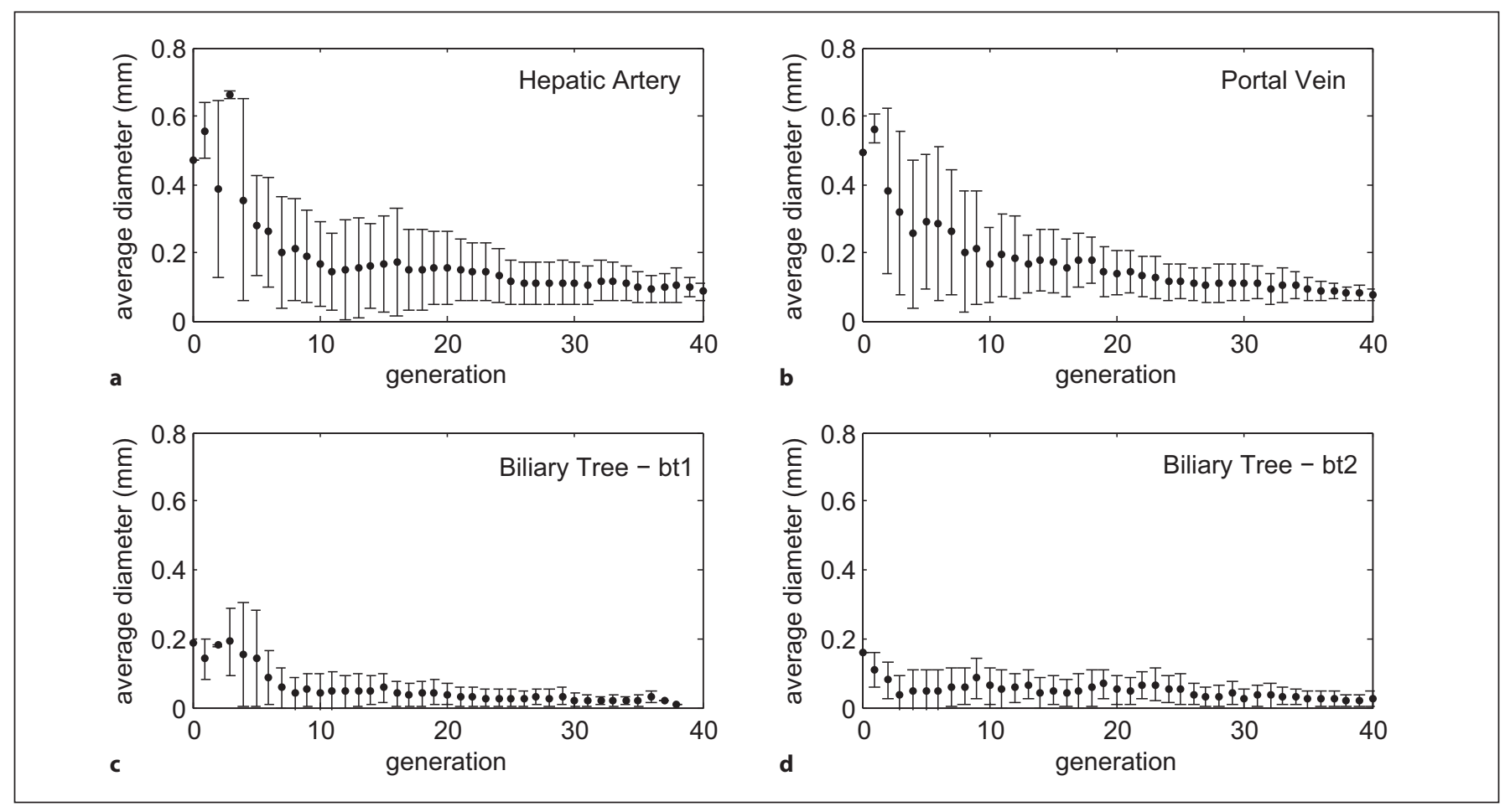

Fig. 4. Average vessel segment diameter at different generations of the hepatic artery (a), the portal vein (b), the biliary tree specimen $b t 1$ (c), and the biliary tree specimen $b t 2(\mathbf{d})$. The error bars represent the standard deviation of the vessel diameters found within each generation.

specimen $b t 1$ (fig. $4 \mathrm{c}$ ), and the biliary tree specimen $b t 2$ (fig. 4d). The standard deviation of the mean is also shown. The behavior is visually similar to that predicted by Murray [1926]. Figure 5 displays the data for interbranch segment length for all specimens (same specimen arrangement as in fig. 4). Significantly more scatter is observed, but a general trend towards a decrease in vessel segment length is also apparent. Figure 6 displays the number of interbranch segments found at each generation for all specimens.

The length/diameter ratio is a critical factor in determining both the relevance of Poiseuille flow to the vascular structures, and also whether local shear stress is a factor in determining branch segment diameters [LaBarbera, 1995]. The average length/diameter ratio ( \pm standard deviation) is $4.85 \pm 3.78$ for the hepatic artery, 5.11 \pm 4.15 for the portal vein, $28.17 \pm 26.71$ for the bile duct $b t 1$, and $25.1 \pm 24.48$ for the bile duct $b t 2$.

The nondimensional diameters (see Eq. 3) for the larger branch segment versus asymmetry ratio are shown in figure 7 for the hepatic artery (fig. 7a), the portal vein (fig. $7 \mathrm{~b}$ ), the biliary tree specimen $b t 1$ (fig. $7 \mathrm{c}$ ), and the biliary tree specimen $b t 2$ (fig. $7 \mathrm{~d}$ ). The nondimensional diameter for the smaller branch segment versus asymmetry ratio are shown in figure 8 , and the area ratio at an arterial bifurcation versus the asymmetry ratio for each sample is shown in figure 9 .

The junction exponent was found to be 2.97 for the hepatic artery, 2.94 for the portal vein, 1.65 for $b t 1$, and 1.84 for $b t 2$. How these values were determined is conveyed in figure 10. Using the data of interbranch segment diameters and their interconnectivity, the relative error was computed by Eq. 5 for $k=1,2,3$ and 4 at each junction of the different vascular trees. The median error value for each $k$ value was then plotted as shown [i.e., $e(k)$ ]. Thus, each vascular tree has four data points (corresponding to their relative errors at each $k$ value). A linear fit to the individual vascular tree's data has a y-intercept that corresponds to the junction exponent which best matches the vessel tree data. In terms of the relative power requirement ( $\psi$ in Eq. 7$)$, the hepatic artery is within $4.3 \%$, the portal vein is within $4.0 \%$, the biliary tree $b t 1$ is within $20.2 \%$, and $b t 2$ is within $16.6 \%$ of the optimal configuration predicted by Murray [1926].

Plots of the larger-diameter nondimensional diameter for the hypothetical model vasculature (the theoretical 

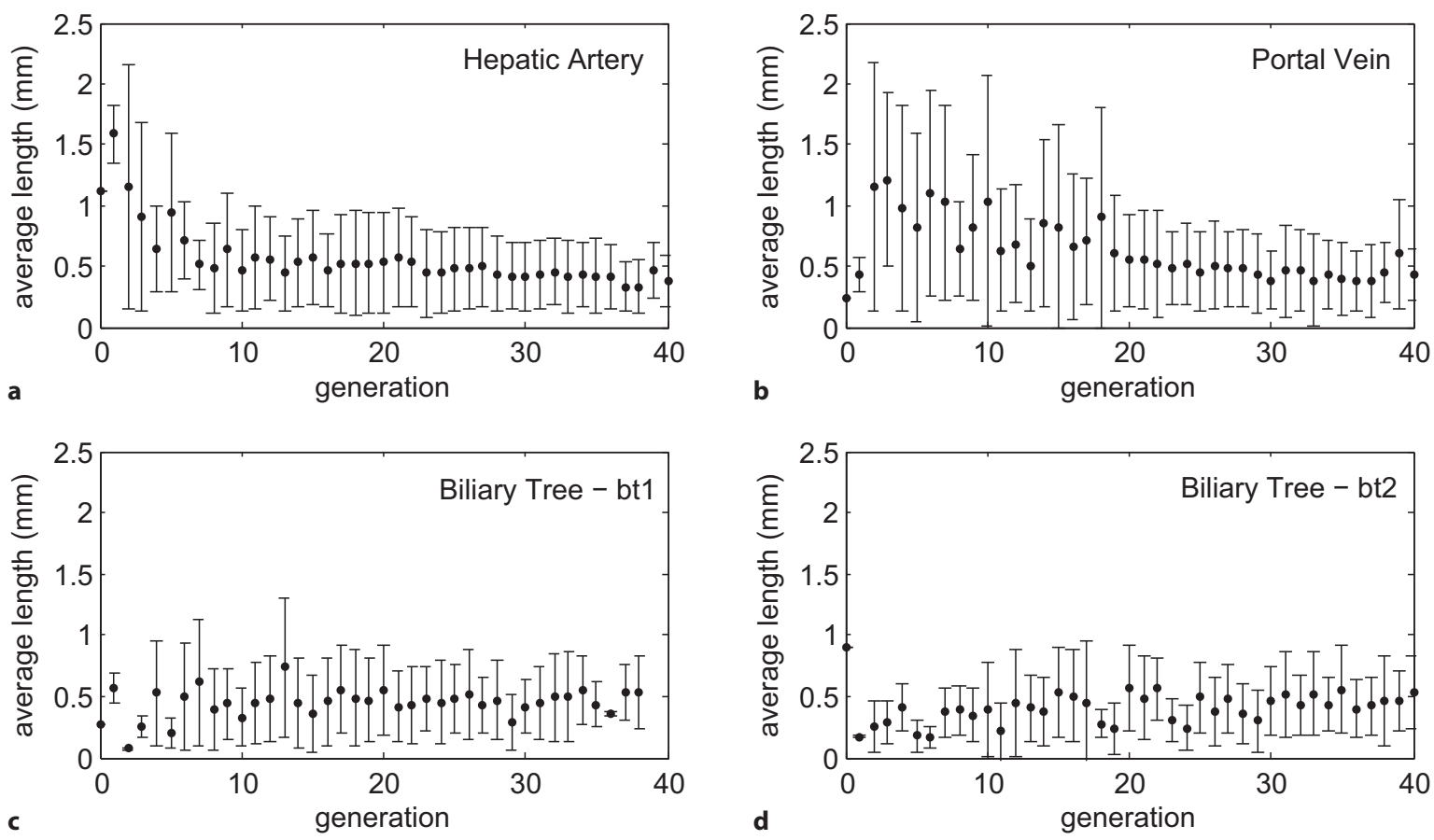

Fig. 5. Interbranch segment lengths in the tree structure of the hepatic artery (a), the portal vein (b), the biliary tree specimen $b t 1(\mathbf{c})$, and the biliary tree specimen $b t 2(\mathbf{d})$. The error bars represent the standard deviation of the vessel lengths found within each generation.
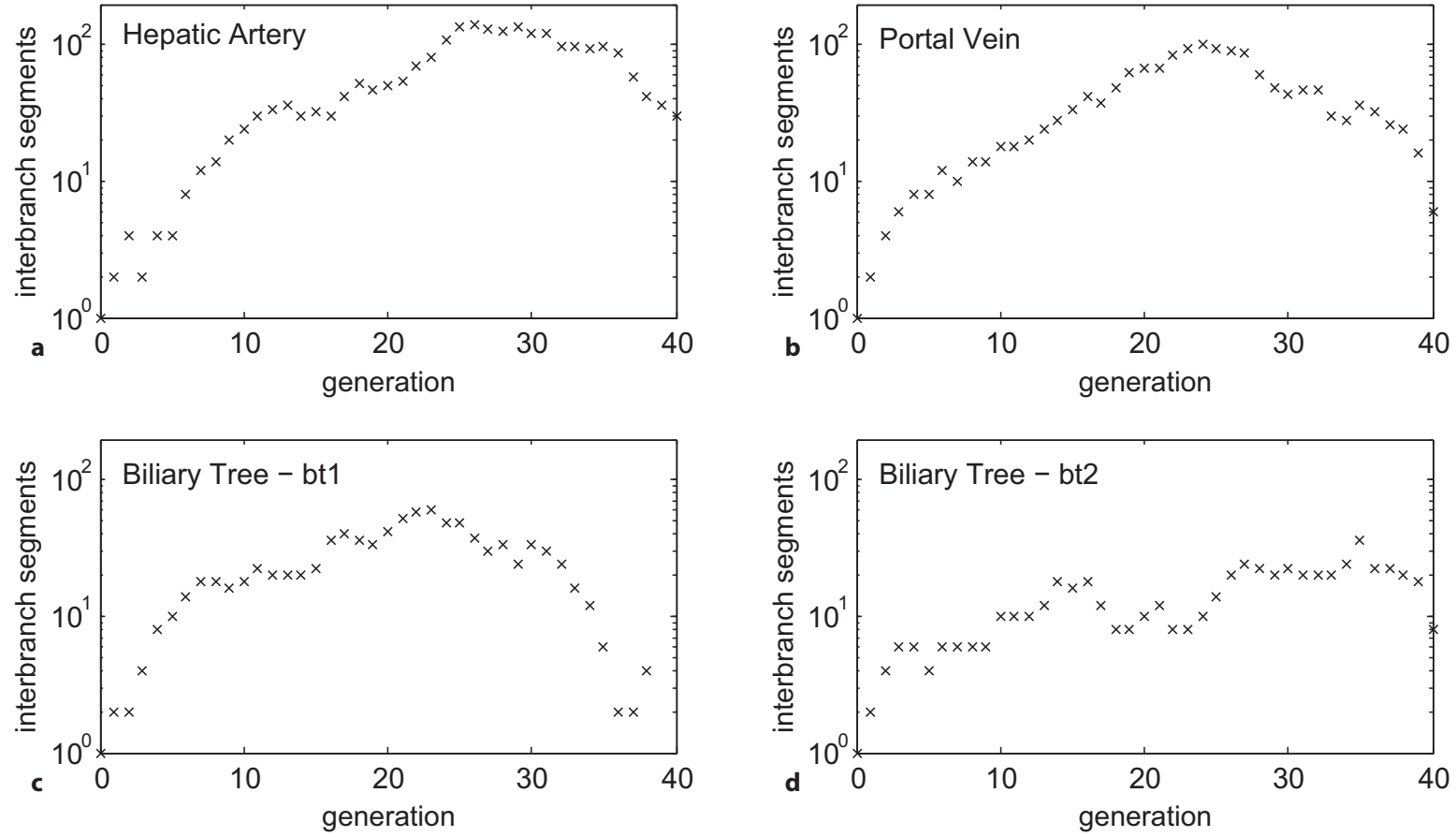

Fig. 6. Number of interbranch segments at each generation for the four specimens. The ordinate axis is here plotted on a logarithmic scale. 


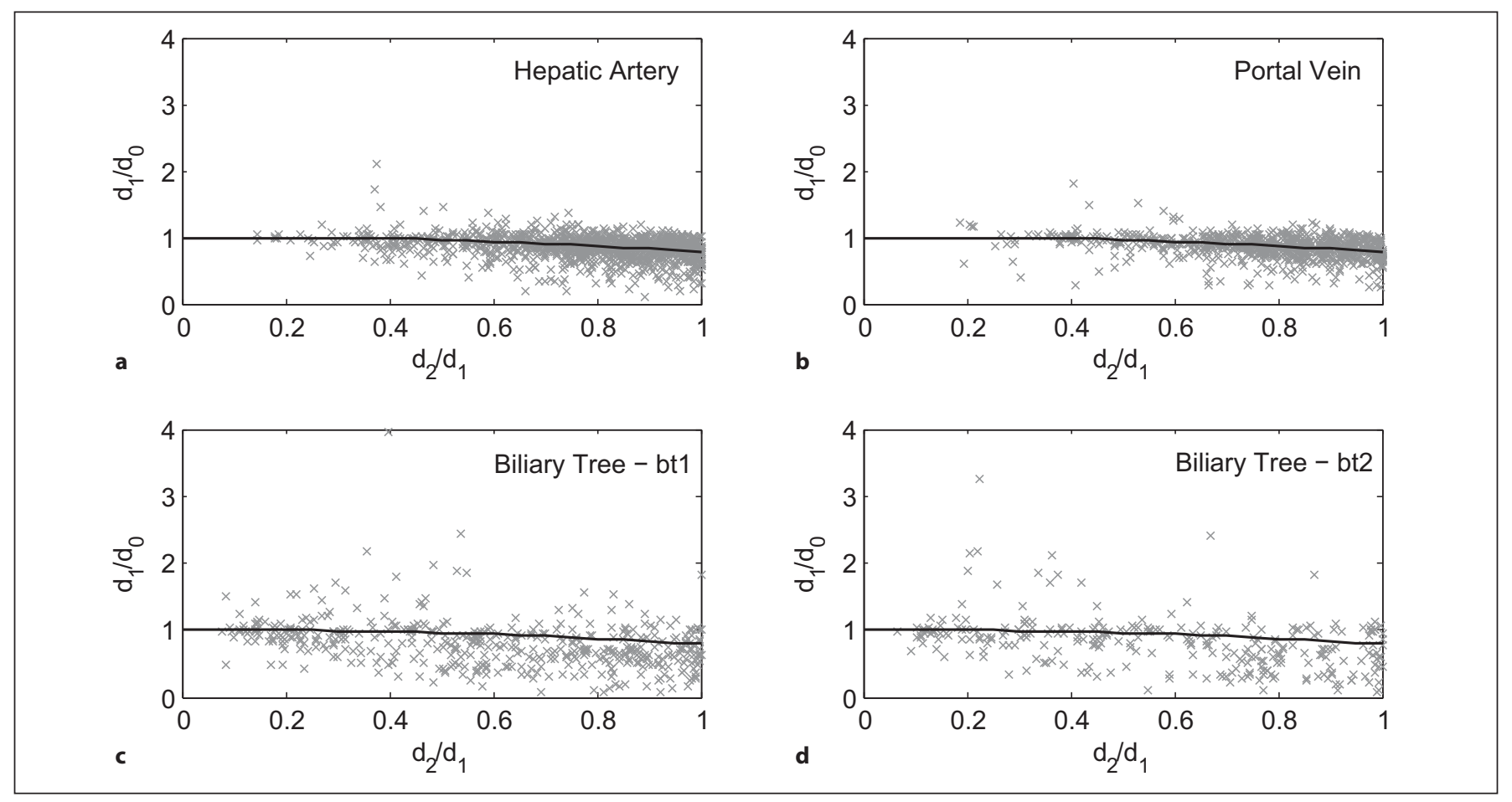

Fig. 7. Nondimensional diameters of the larger branch segments' asymmetry ratio in the tree structure of the hepatic artery (a), the portal vein (b), the biliary tree specimen $b t 1$ (c), and the biliary tree specimen $b t 2$ (d). The result for an ideal tree following a 'cube' law (solid black line) is also presented.

equivalent of the experimental data presented in fig. 6) are shown in figure 11 . The variability is set at $5 \%$ (fig. 11a), $10 \%$ (fig. 11b), $20 \%$ (fig. 11c), and 40\% (fig. 11d). Also, note the different scale for $40 \%$ variability. In each plot the results for an ideal tree following a 'square' law (dots) as well as a 'cube' law (solid black line) are shown. The line representing the tree following a 'cube' law is essentially our model's initial state, before incorporating variability.

\section{Discussion}

Measuring vascular casts is historically the method used for measuring vascular branching networks [Suwa et al., 1963; Zamir, 1996; Kalsho and Kassab, 2004]. However, such methods typically lose information pertaining to the interconnectivity of branch segments. This is due to the fact that often the casts are physically broken in order to measure the physical dimensions of interbranch segments, and statistical analysis approaches are then used to say something general about the characteristics of the vascular bed [Spaan, 1991]. The method of optical measurement of an intact vascular cast is very time consuming, labor intensive, and highly inefficient. In the work of Zamir [1996], more than 1 year was required for measurements of the vascular tree from a right coronary artery consisting of 4,531 interbranch segments. At that time, image analysis methods were found inadequate in terms of accuracy, and the technical problem of automatically evaluating the hierarchical structure of the vascular tree had not been solved. Finally, the method of casting is of course not applicable to the tissue of a living specimen due to its destructive nature.

Using the automated analysis of micro-CT images, instead of optical measurements of a cast, saves time and allows analysis of highly complex tree structures. For example, the hepatic artery analyzed in this study is composed of more than 2,000 interbranch segments, and the interbranch relationships, measurement of interbranch segment lengths and diameters were performed automatically in minutes, as opposed to months. More important, using micro-CT images of in situ, opacified microvasculature, instead of an isolated cast, means that the measurement technique can be easily extended to in vivo analysis. 


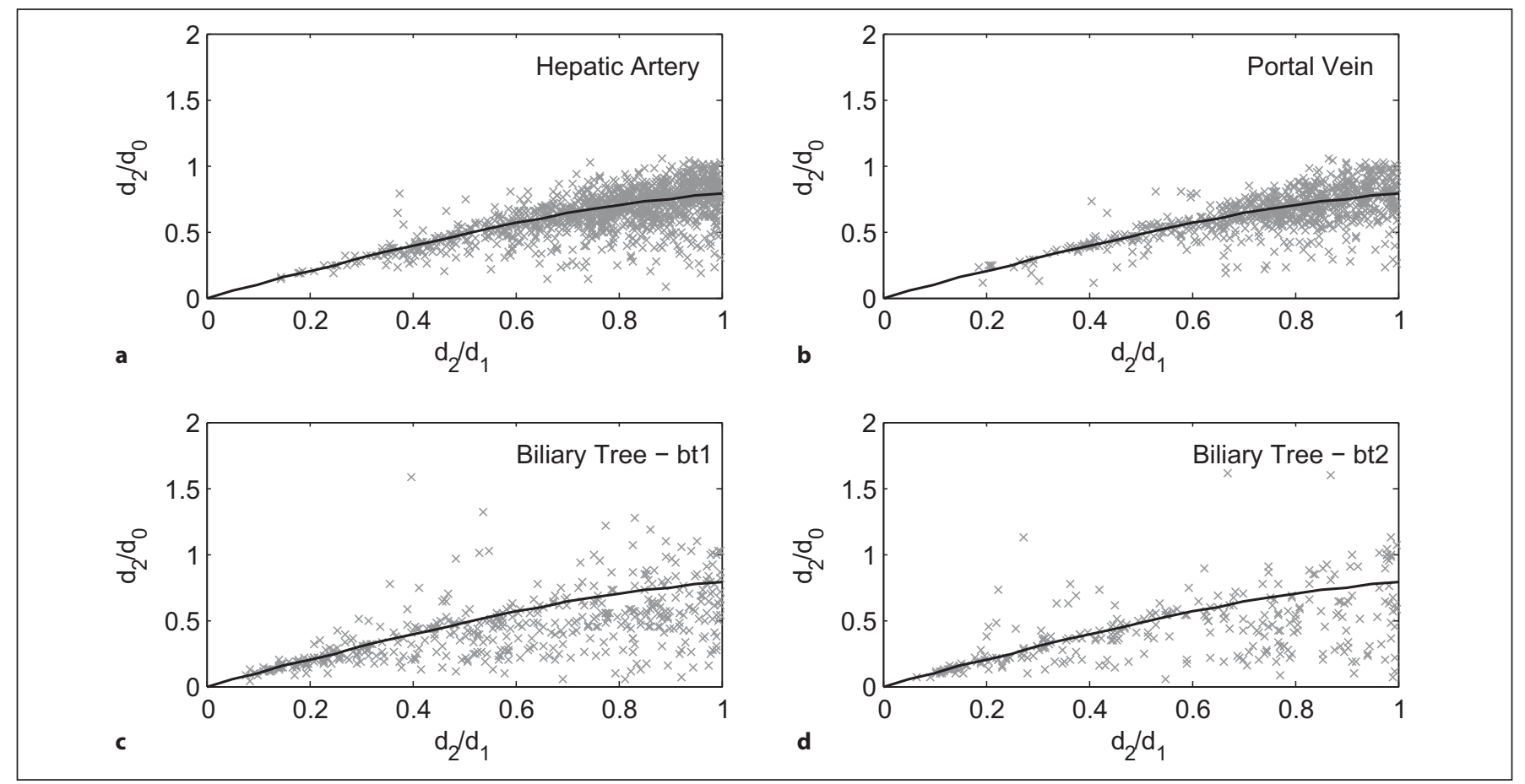

Fig. 8. Nondimensional diameters of the smaller branch segments' asymmetry ratio in the tree structure of the hepatic artery (a), the portal vein (b), the biliary tree specimen $b t 1$ (c), and the biliary tree specimen $b t 2$ (d). The result for an ideal tree following a 'cube' law (solid black line) is also presented.

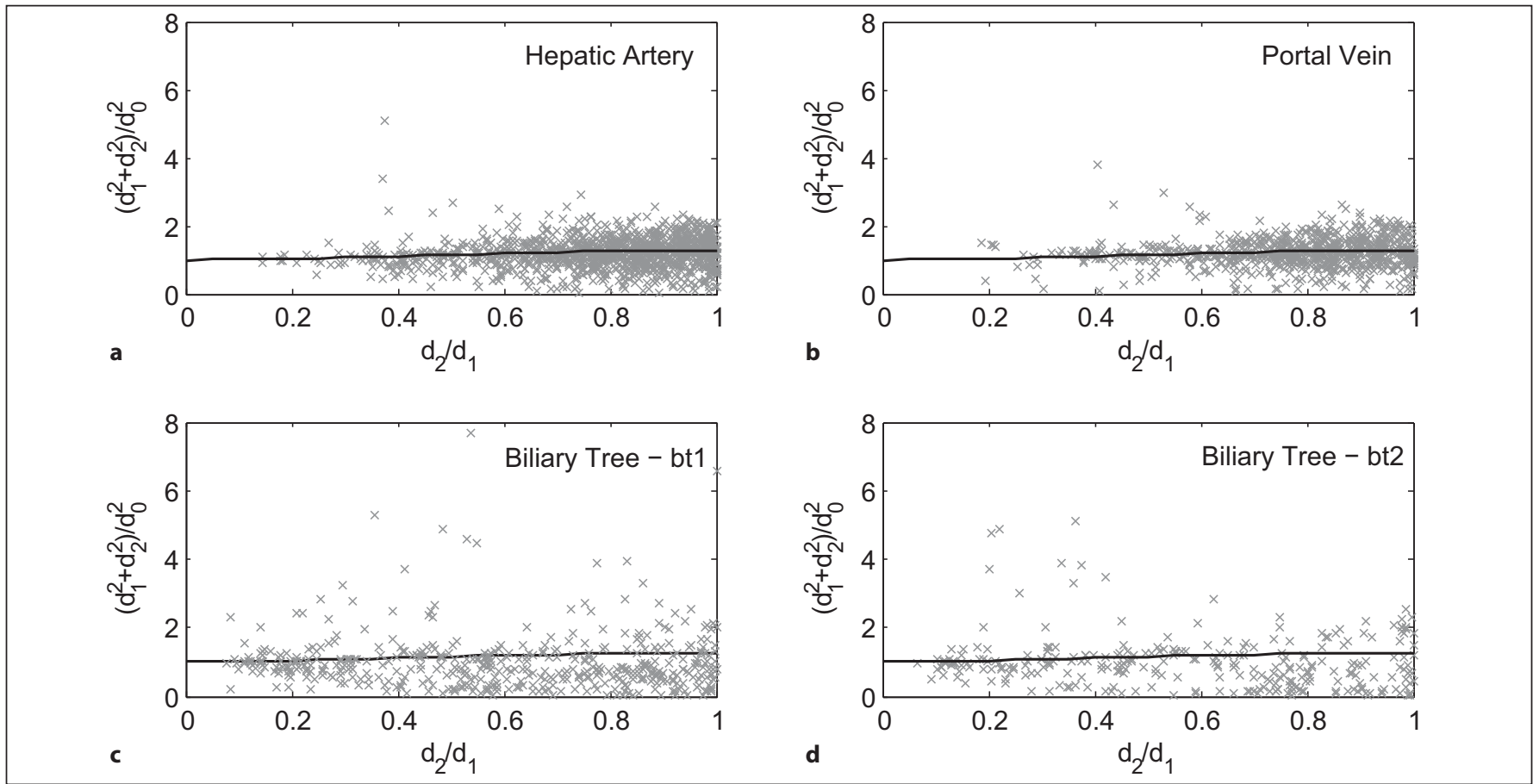

Fig. 9. Area ratios at arterial bifurcations versus the asymmetry ratios in the tree structures of the hepatic artery (a), the portal vein (b), the biliary tree specimen $b t 1$ (c), and the biliary tree specimen $b t 2(\mathbf{d})$. The result for an ideal tree following a 'cube' law (solid black line) is also presented. 
Fig. 10. To determine the junction exponent for each specimen the relative error was computed by Eq. 5 for $k=1,2,3$ and 4 at each junction of the different vascular trees. The median error value for each $k$ value was then plotted as shown. Thus, each vascular tree has four data points (corresponding to their relative errors at each $k$ value). A linear fit to the individual vascular tree's data has a y-intercept that corresponds to the junction exponent which best matches the vessel tree data. As shown, the hepatic artery and portal vein are best represented as vascular structures which adhere to Murray's cube law. The two biliary trees, on the other hand, follow a square law. $\mathrm{HA}=$ Hepatic artery; $\mathrm{PV}=$ portal vein; BT1 and BT2 = biliary tree 1 and 2.
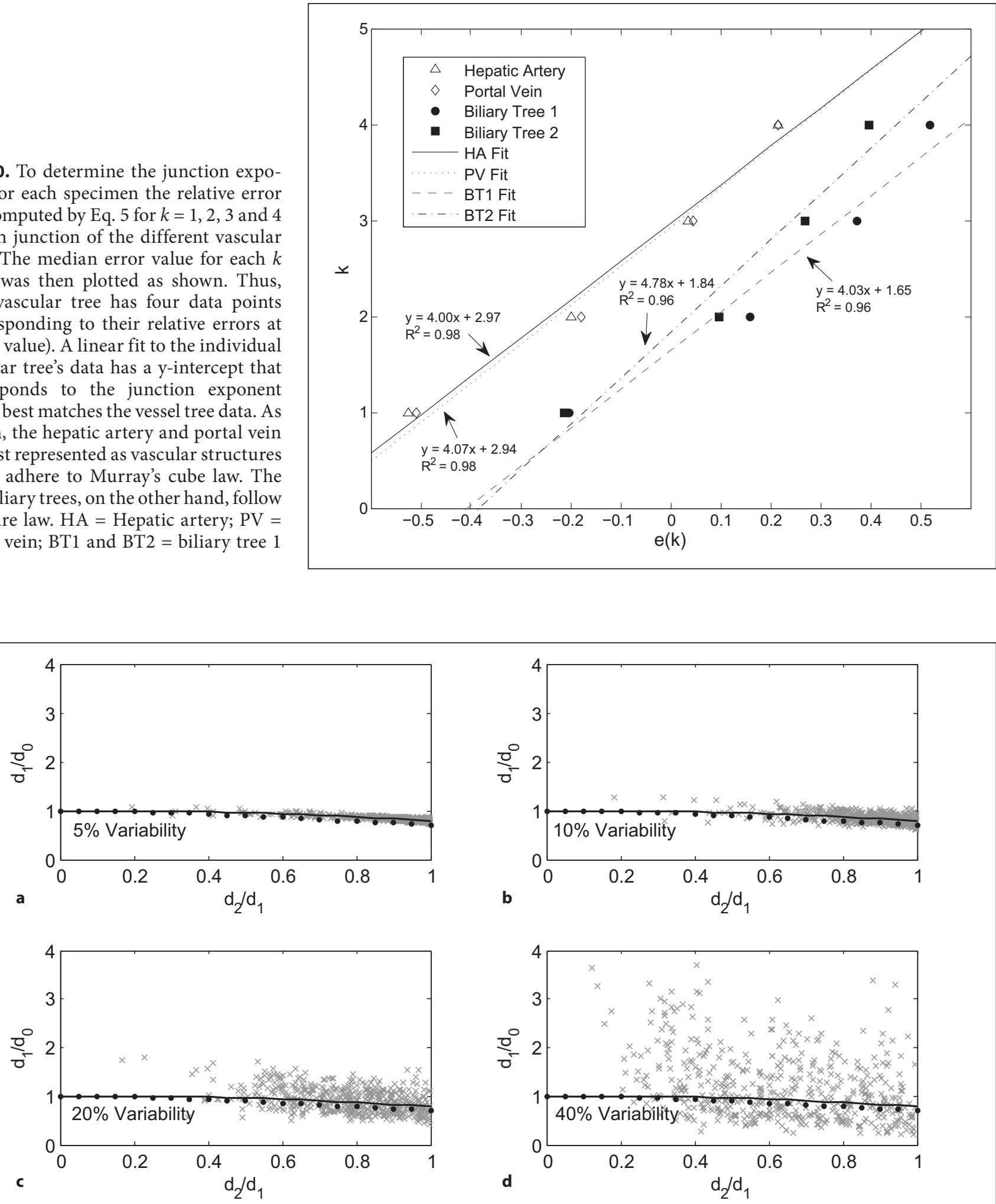

Fig. 11. Theoretical larger branch nondimensional diameter with imposed $5 \%$ variability (a), $10 \%$ variability (b), $20 \%$ variability (c), and $40 \%$ variability (d). Also shown are the result for an ideal tree following a 'square' law (dots) and a 'cube' law (solid black line). 
A methodology issue in the present study is that the different vascular trees were obtained from different rat specimens, although each vascular structure was composed of approximately the same number of generations. Ideally all three vessels should have been injected in a single liver. However, in addition to the technical difficulties involved in sample preparation, this would likely result in difficulty in differentiating small diameter branch segments in the reconstructed image due to their close proximity to each other (i.e., 'overlapping' of neighboring vessel gray scales due to blurring).

The results in general show some important differences between the trees' vascular branching geometries: most notably, the strong adherence to Murray's cube law by the hepatic artery and portal vein, and the large deviation from Murray's cube law by the biliary tree. Since the flow rate through a vascular tree must be the same at all levels of the tree, the square law, whereby the crosssectional area available to the flow is the same at all levels of the tree, implies that the average velocity of the fluid is also the same at all levels of the tree. In the systemic arterial tree this situation is not supportable since the high velocity of blood in the aorta must be reduced by a factor of about 1,000 by the time it reached the capillaries [Zamir, 2005]. Furthermore, since the shear stress at the endothelial layer of a vessel segment in Poiseuille flow is proportional to the flow rate over the cube of the vessel diameter, the square law also implies that the shear stress becomes increasingly higher towards the peripheral end of the tree as vessel diameters become smaller [Zamir, 2005]. Again, this situation is not supportable in the arterial tree where the shear stress acting on endothelial tissue must be the same. Thus, our finding that the square law is supported in the biliary tree suggests that the optimality considerations of Murray's law are of little consequence in the biliary tree. Thus the characteristics of the flow in the biliary tree are likely closer to those of a low velocity bolus flow rather than Poiseuille flow.

We suspect that the main driving force within the biliary tree is simply that of bile entering at the peripheral end of the tree and pushing/displacing fluid forward, evidently at very low speed, towards the central levels and ultimately the exit end of the tree. These findings imply that the histological makeup and therefore the pathophysiology of biliary tree vasculature is likely very different from that of vasculature within the systemic arterial tree. Recent findings have shown morphological heterogeneity of the biliary epithelium, as well as a wide range of different pathophysiological responses by differentsized bile ducts [Glaser et al., 2006].

Relating Function to Branching

Geometry: A Micro-CT Study
The strength of this study's method for measuring the junction exponent (as opposed to a minimization type approach [Op Den Buijs et al., 2006]) is that the collective behavior of the vascular tree's range of junction exponents can be determined while still including data in which one of the branch segments may be larger than the parent segment (in which case a minimization approach does not have a unique solution). The range of exponents found in the literature range from 1.0 up to 5.0 [Zamir et al., 1992], but most frequently lie between 2.0 and 3.0 [Kassab, 2006]. In addition, the energy cost ( $\psi$ from Eq. 6) calculated for this study's vascular trees are within the cited range of 2-24\% [Sherman et al., 1989]. Intuitively it makes sense that the biliary tree would not need to adhere to Murray's law as do the other vascular structures. The low-flow, low-pressure environment would require little investment in materials needed to maintain the system (such as little smooth muscle to contain the pressure).

A notable shortcoming of Murray's law is that there is an unrealistic idealization for fluid flow in the vicinity of vessel junctions. Also, real vascular structures are not actually composed of cylindrical segments carrying parabolic Poiseuille flow profiles. For one, the flow is necessarily altered at branching junctions, and second, Poiseuille flow will only be reestablished downstream some distance from a bifurcation: $10-80$ vessel diameters downstream [LaBarbera, 1995].

The much larger length/diameter ratio of the biliary tree is particularly interesting. A large length/diameter ratio, at least above a value of 10 , is necessary for fully developed Poiseuille flow, yet the vascular trees which closely mirror the ideal Murray-type tree (the hepatic artery and portal vein as demonstrated by fig. 6,7$)$ do not frequently satisfy this criterion. Since measurement of interbranch segment lengths have largely been ignored, data related to this geometric property are scarce. It appears probable that the biliary tree, unlike the hepatic artery and portal vein, favors more tortuous and lengthy pathways, which could result from the biliary tree retaining fluids when not discharging bile to the intestine (a function somewhat compensated for by the human gallbladder). This additional criterion would result in a distinct deviation from Murray's cube law. The median volume ratio [calculating $V_{0} /\left(V_{1}+V_{2}\right)$ at each bifurcation] was found to be exactly 1.0 for the bile ducts, whereas the volume ratio of the hepatic artery and portal vein was 0.7 . This suggests that the biliary tree more closely adheres to local optimization criteria that require the total vessel volume to remain constant between a parent segment and its branches (recall that the branches actually flow into the parent segment).

Cells Tissues Organs 2011;194:431-442 
From the theoretical analysis of variability, we conclude that neither variability in an ideal Murray-type tree, nor noise/measurement error would explain the observed 'square' law behavior of the biliary tree in our experimental data. Thus, the question is whether the hepatic artery and portal vein measurements are biased. Based on the plots of the normalized diameter (comparing fig. 7-11), our data is consistent with a $10 \%$ variability. In our theoretical analysis, this resulted in a change in the junction exponent from 3.0 to 3.15. Thus, variability (derived from either measurement error, or variability within the vascular trees) would not cause the differences observed when comparing the hepatic artery and portal vein to the biliary trees. In addition, variability (actual deviations from Murray-tree-type behavior) could help to explain the large amounts of scatter [Zamir et al., 1992; LaBarbera, 1995; Kassab, 2006] typically found when comparing vascular tree data to those predicted by Murray.

Lastly, it should be noted that vasculature plays a major role in various diseases (e.g., heart disease, tumors, peripheral vascular disease, diabetes) [Sinnatamby, 2006; Standring and Gray, 2008]. Therefore, the ability to characterize geometrical differences in vascular structures performing functionally different roles will also be useful for outlining differences within the same vascular structures in, for example, different states of disease. Such a characterization would likely involve extending the analysis to the computation of local blood flow magnitudes, pressure distributions, and local shear stresses [Yang et al., 2010].

\section{Acknowledgements}

The authors would like to thank Dr. Tetyana Masyuk for use of the micro-CT images of her rat liver samples, Mr. Steven M. Jorgensen for scanning the specimens, Mr. Andrew J. Vercnocke and Ms. Diane R. Eaker for their help with image reconstructions, and Ms. Delories C. Darling for her help in the preparation of this manuscript. This work was supported in part by NIH grant EB000305 and The Mayo Foundation.

\section{References}

-Birnie, J.H., J. Grayson (1952) Observations on temperature distribution and liver blood flow in the rat. J Physiol 116: 189-201.

-Feldkamp, L.A., L.C. Davis, J.W. Kress (1984) Practical cone-beam algorithm. J Opt Soc Am A 1: 612-619.

Glaser, S., H. Francis, S. DeMorrow, G. LeSage, G. Fava, M. Marzioni, J. Venter, G. Alpini (2006) Heterogeneity of the intrahepatic biliary epithelium. World J Gastroenterol 12: 3523-3536.

Glover, G.H., N.J. Pelc (1980) Nonlinear partial volume artifacts in $\mathrm{x}$-ray computed tomography. Med Phys 7: 238-248.

Herman, G.T. (1980) Image Reconstruction from Projections: The Fundamentals of Computerized Tomography. New York, Academic Press.

Jorgensen, S.M., O. Demirkaya, E.L. Ritman (1998) Three-dimensional imaging of vasculature and parenchyma in intact rodent organs with $\mathrm{x}$-ray micro-CT. Am J Physiol Heart Circ Physiol 275: H1103-H1114.

-Kalsho, G., G.S. Kassab (2004) Bifurcation asymmetry of the porcine coronary vasculature and its implications on coronary flow heterogeneity. Am J Physiol Heart Circ Physiol 287: H2493-H2500.

-Kassab, G.S. (2006) Scaling laws of vascular trees: of form and function. Am J Physiol Heart Circ Physiol 290: H894-H903.

Kline, T.L., Y. Dong, M. Zamir, E.L. Ritman (2010a) Erode/dilate analysis of micro-CT images of porcine myocardial microvasculature. Proc SPIE 7626: 762620-1-762620-9.
Kline, T.L., E.L. Ritman (2009) Paths of least flow-resistance: characterization for the optimization of synthetic tissue scaffold design. Proc ISBI 606-609.

Kline, T.L., M. Zamir, E.L. Ritman (2010b) Accuracy of microvascular measurements obtained from micro-CT images. Ann Biomed Eng 38: 2851-2864.

LaBarbera, M. (1995) The design of fluid transport systems: a comparative perspective; in Bevan, J.A., G. Kaley, G.M. Rubanyi (eds): Flow Dependent Regulation of Vascular Function. Oxford, Oxford University Press, pp 3-27.

Lee, J., P. Beighley, E.L. Ritman, N. Smith (2007) Automatic segmentation of 3D micro-CT coronary vascular images. Med Image Anal 11: 630-647.

Masyuk, T.V., E.L. Ritman, N.F. LaRusso (2003) Hepatic artery and portal vein remodeling in rat liver vascular response to selective cholangiocyte proliferation. Am J Pathol 162: $1175-1182$.

Murray, C.D. (1926) The physiological principle of minimum work. I. The vascular system and the cost of blood volume. Proc Natl Acad Sci USA 12: 207-214.

Op Den Buijs, J., Z. Bajzer, E.L. Ritman (2006) Branching morphology of the rat hepatic portal vein tree: a micro-CT study. Ann Biomed Eng 34: 1420-1428.

Robb, R.A., D.P. Hanson, M.C. Stacy (1989) ANALYZE: a comprehensive, operator interactive software package for multidimensional medical image display and analysis. Comput Med Image Graph 13: 433-454.
-Sherman, T.F., A.S. Popel, A. Koller, P.C. Johnson (1989) The cost of departure from optimal radii in microvascular networks. J Theor Biol 136: 245-265.

Sinnatamby, C.S. (2006) Last's Anatomy: Regional and Applied. Edinburgh, Churchill Livingstone.

Spaan, J.A.E. (1991) Coronary Blood Flow. Dordrecht, Kluwer Academic Publishers.

Standring, S., H. Gray (2008) Gray's Anatomy: The Anatomical Basis of Clinical Practice. Edinburgh, Churchill Livingstone.

Suwa, N., T. Takahashi, H. Fukasawa, Y. Sasaki (1963) Estimation of intravascular blood pressure gradient by mathematical analysis of arterial casts. Tohoku J Exp Med 79: 168-198.

-Yang, J., L.X. Yu, M.Y. Rennie, J.G. Sled, R.M. Henkelman (2010) Comparative structural and hemodynamic analysis of vascular trees. Am J Physiol Heart Circ Physiol 298: H1249H1259.

Zamir, M. (1996) Tree structure and branching characteristics of the right coronary artery in a right-dominant human heart. Can J Cardiol 12: 593-599.

Zamir, M. (1998) Mechanics of blood supply to the heart: wave reflection effects in a right coronary artery. Proc Biol Soc 265: 439-444.

Zamir, M. (2005) The Physics of Coronary Blood Flow. New York, Springer Science and Business Media.

Zamir, M., P. Sinclair, T.H. Wannacott (1992) Relation between diameter and flow in major branches of the arch of the aorta. J Biomech 25: 1303-1310. 